 \\ Корпусные методы исследования в решении переводческих задач
}

\author{
Абдульманова А. Х., Куликова М. Н., Лекомцева И. А.
}

Аннотация. Цель исследования - сопоставительный анализ модусных наречий в английском языке на основе корпусных данных. При прескриптивном подходе к переводу ключевое значение представляет способность выявлять закономерности использования языковых средств в речи на языке перевода. Одним из способов выявления таких закономерностей могут быть корпусные методы исследования в переводоведении. Научная новизна исследования заключается в том, что корпусные методы исследования впервые были использованы в практике перевода на неродной язык в аспекте прескриптивного переводоведения. В результате выявлены типичные, регулярные модели употребления данных наречий в различных функциональных стилях речи.

\section{EN Corpus Research Methods in Solving Translation Problems}

\begin{abstract}
Abdulmanova A. K., Kulikova M. N., Lekomtseva I. A.
Abstract. The paper aims to carry out a comparative analysis of modus adverbs in the English language using corpus data. Under a prescriptive approach to translation, the ability to identify patterns in the use of linguistic means in speech in the target language is of key importance. One of the ways to identify such patterns can be corpus research methods in translation studies. Scientific novelty of the work lies in the fact that corpus research methods have been applied in the practice of translating into a non-mother tongue in the aspect of prescriptive translation studies for the first time. As a result, the authors have identified typical, recurring models in the use of the considered adverbs in various functional speech styles.
\end{abstract}

\section{Введение}

Актуальность темы исследования обусловлена тем, что инкорпорирование новейших технологий в науку позволяет по-новому решать ее фундаментальные задачи. В теории и практике перевода активно используются методы корпусной лингвистики с целью выявления закономерностей использования языковых единиц в речи, что особенно актуально при переводе на неродной язык. Такой подход представляет собой эвристическую ценность для перевода, особенно для практики перевода и оценки качества перевода, поскольку при переводе первостепенную важность представляет умение определять соответствия не столько между языковыми системами, сколько между употреблениями языковых единиц в речи (Швейцер, 1993), а оценка перевода становится менее субъективной (Bowker, 2000). Знание типичных, регулярных моделей словоупотребления с точки зрения закономерностей функционирования языка в речи отражает язык в его предречевой готовности и оптимизирует эвристический поиск при переводе.

Использование корпусных методов при переводе активно изучается в последнее время как в отечественных (Захаров, Богданова 2020), так и в зарубежных исследованиях. Так, например, корпусные методы исследования перевода были привнесены в область аудиовизуального перевода (Bruti, 2020). Корпусные исследования также широко представлены в области юридического перевода (Corpus-Based Research..., 2019). Были проведены корпусные исследования в переводоведении в Венгрии (Edina, Götz, Pataky, 2018). Особую ценность представляют исследования на стыке нескольких областей знания: переводоведения, корпусной лингвистики и когнитивной лингвистики (Garcia, 2021). Также рассматривается применение конкретных возможностей корпусных менеджеров в дескриптивном переводоведении (Buts, Jones, 2021).

Такой интегративный подход в области сопоставительно-переводческих исследований широко представлен в современных работах за рубежом, однако в России ему уделено внимание всего в нескольких публикациях.

Широта и разнообразие работ в области применения корпусных методов в переводоведении могут служить подтверждением перспективности исследований в данной области. Тем не менее, многие вопросы остаются нерешенными, в том числе: могут ли корпусные исследования внести вклад в прескриптивное 
переводоведение, например с точки зрения определения возможных вариантных соответствий в принимающем языке, а также предоставить данные о возможных соответствиях с точки зрения их сходства и различия как на уровне системы языка, так и на уровне закономерностей их использования в речи?

Выбор модусных наречий в качестве предмета исследования представляется целесообразным, поскольку данные наречия относятся к широкой семантической категории модальности возможности, которая реализуется во всех функциональных стилях независимо от тематики текстов, что, в свою очередь, позволяет привлечь для анализа весь материал корпусов, а не ограничиться какой-либо тематикой или функциональным стилем речи. Результаты исследования могут применяться в практике перевода, особенно на втором этапе переводческого семиозиса, т.е. при перевыражении подлинника средствами принимающего языка, на этапе выбора языковых средств для выражения инварианта, т.е. в данном случае категории модальности.

Для достижения поставленной цели решим следующие задачи:

- определим устойчивые системные черты сходства и различия между рассматриваемыми наречиями на основе данных толковых словарей;

- проанализируем корпусные данные, которые выявляют закономерности использования рассматриваемых наречий в речи, а именно: какие значения ассоциируются с этими словами; какова частотность этих слов по отношению к друг другу в разных регистрах и функциональных стилях речи; какие слова обычно встречаются вместе с рассматриваемыми словами и каково распределение этих сочетаемостных возможностей в разных регистрах.

Решение поставленных задач определяет выбор методов исследования: использованы метод семного анализа, корпусные методы исследования и сопоставительный метод.

Теоретической базой исследования послужили публикации зарубежных авторов, в которых рассматриваются возможности и перспективы инкорпорирования корпусных методов в переводоведение и сопоставительное языкознание (Doval, Sánchez Nieto, 2019; Mikhailov, Cooper, 2016; Ji, Oakes, Defeng et al., 2018; Pastor, Seghiri, 2016; Oaks, Ji, 2012; De Sutter, Cappelle, De Clercq et al., 2018).

Практическая значимость исследования заключается в том, что выявленные закономерности использования языковых единиц в речи и их систематизация обеспечивают успешность переводческой деятельности на втором этапе переводческого семиозиса, т.е. в процессе выбора языковых средств при перевыражении инварианта в принимающем языке, благодаря тому, что практические результаты исследования могут внести вклад в представление языка в его предречевой готовности. Такой подход важен, поскольку он способствует видению переводческого процесса как речепорождающей деятельности по заданной в оригинале программе, а не трансформационно-манипулятивной деятельности с единицами исходного языка (Крюков, 1988; Крюков, 1989). Полученные результаты можно использовать в процессе преподавания дисциплин по письменному переводу с русского языка на английский язык.

\section{Основная часть}

Представим данные толкового словаря Oxford English Dictionary (2021) в Таблице 1.

Таблица 1. Словарные определения модусных наречий

\begin{tabular}{|c|c|c|c|c|c|c|c|}
\hline $\begin{array}{l}\text { Probably/ } \\
\text { Вероятно }\end{array}$ & $\begin{array}{l}\text { Possibly/ } \\
\text { Возможно }\end{array}$ & $\begin{array}{l}\text { Perhaps/ } \\
\text { Возможно }\end{array}$ & $\begin{array}{c}\text { Мaybe/ } \\
\text { Может быть }\end{array}$ & $\begin{array}{l}\text { Presumably/ } \\
\text { Предполо- } \\
\text { жительно }\end{array}$ & $\begin{array}{l}\text { Conceivably/ } \\
\text { Возможно }\end{array}$ & $\begin{array}{c}\text { Apparently/ } \\
\text { По-видимому }\end{array}$ & $\begin{array}{l}\text { Plausibly/ } \\
\text { Вероятно }\end{array}$ \\
\hline \multirow[t]{2}{*}{$\begin{array}{l}\text { Sentence adverb } \\
\text { Almost certain- } \\
\text { ly; as far as one } \\
\text { knows or can } \\
\text { tell. / Почти } \\
\text { наверняка; } \\
\text { насколько } \\
\text { известно } \\
\text { или можно } \\
\text { сказать. }\end{array}$} & \begin{tabular}{|} 
1. Sentence \\
adverb \\
Perhaps (used \\
to indicate doubt \\
or hesitancy). / \\
Возможно \\
(используется \\
для обозначе- \\
ния сомнений \\
или нереши- \\
тельности).
\end{tabular} & $\begin{array}{c}\text { Adverb } \\
\text { Used to express } \\
\text { uncertainty or } \\
\text { possibility./ } \\
\text { Используется } \\
\text { для выражения } \\
\text { неуверенности } \\
\text { или возмож- } \\
\text { ности. }\end{array}$ & $\begin{array}{c}\text { Adverb } \\
\text { Perhaps; роssi- } \\
\text { bly. / Возможно. }\end{array}$ & $\begin{array}{c}\text { Sentence adverb } \\
\text { Used to convey } \\
\text { that what is } \\
\text { asserted is very } \\
\text { likely though } \\
\text { not known } \\
\text { for certain./ } \\
\text { Используется } \\
\text { для обозначе- } \\
\text { ния того, } \\
\text { что утвержда- } \\
\text { ется, очень } \\
\text { вероятно, хотя } \\
\text { и не известно } \\
\text { наверняка. }\end{array}$ & $\begin{array}{l}\text { Sentence adverb } \\
\text { It is conceivable } \\
\text { or imaginable } \\
\text { that. / } \\
\text { Возможно } \\
\text { или вообразимо, } \\
\text { что. }\end{array}$ & $\begin{array}{l}\text { Sentence adverb } \\
\text { As far as one } \\
\text { knows or can } \\
\text { see. / Насколько } \\
\text { известно } \\
\text { или видно. }\end{array}$ & \begin{tabular}{|c|} 
Adverb \\
In a way that \\
seems reasona- \\
ble or probable. / \\
Таким образом, \\
который \\
кажется \\
разумным \\
или вероятным.
\end{tabular} \\
\hline & $\begin{array}{l}\text { 2. Usually } \\
\text { with modal } \\
\text { In accordance } \\
\text { with what is } \\
\text { likely or achiev- } \\
\text { able./ } \\
\text { В соответствии } \\
\text { с тем, что ве- } \\
\text { роятно или до- } \\
\text { стижимо. }\end{array}$ & & & & & & \\
\hline
\end{tabular}


Зачастую в определениях этих слов присутствует значительное количество повторяющихся семантических компонентов. Так, например, пересекаются значения слов probably и apparently с общим семантическим компонентом [as far as one knows or can tell]; слова perhaps, possibly и тауbе с общим семантическим компонентом [perhaps]; plausibly и probably с общим семантическим компонентом [probable]. На основании словарных данных иногда можно выявить степень вероятности, которую выражает наречие. Однако словарь не предоставляет данные о том, как слово функционирует в речи, какие закономерности, модели употребления типичны для данного слова.

Обратимся к анализу корпусных данных. Для исследования был использован Британский национальный корпус BNCWeb at Lancaster University (BNC, 2021). Начнем исследование с анализа коллокатов рассматриваемых слов. С помощью функции стандартного запроса (Standard Query) были проанализированы левые и правые коллокаты, непосредственно предшествующие или следующие за ключевым словом соответственно, а также случаи сочетаемостных возможностей данных наречий с той или иной частью речи (collocations on POS-tags) с указанием семантического класса. Для сравнения была использована функция статистического показателя устойчивости сочетания слов log-likelihood value, т.е. значение, которое показывает, является ли статистически значимой большая частотность коллокации в одном корпусе по сравнению с частотностью этой коллокации в другом корпусе. Для анализа был выбран подкорпус письменных текстов Британского национального корпуса. Были проанализированы первые пять результатов для каждого наречия.

Приведем значения нормированных частот употребления наречия probably в разных функциональных стилях: художественная литература (268), научная литература (243), газетно-информационный стиль (165). Выявлено, что для наречия probably наиболее частотны левые коллокаты (примерно 85-90\%). На первом месте стоят левые коллокаты, выраженные модальными глаголами (modal auxiliary verb (e.g. will, would, can, could, 'll, 'd)) (87\%); log-likelihood value - 16986.5119; пример модели употребления: The best thing would probably be just to walk around to keep warm (BNC, 2021). / Лучше всего было бы просто прогуляться, чтобы согреться (здесь и далее перевод был выполнен авторами статьи. - А. А., М. К.). Наиболее частотным модальным глаголом является глагол would. Следующим наиболее частотным левым коллокатом является глагол to be в 3 лице ед.ч. (the -s form of the verb BE: is, 's); log-likelihood value - 7769.8267; пример модели употребления: That is probably the most important question the panel are asking themselves (BNC, 2021). / Это, наверное, самый важный вопрос, который задает себе группа. На третьем месте стоит глагол tо bе в прошедшем времени (the past tense forms of the verb BE: was and were); log-likelihood value - 4415.3647; пример модели употребления: She was probably just seeking attention (BNC, 2021). / Вероятно, она просто искала внимания. На четвертом месте - глагол to be в форме инфинитива (the infinitive form of the verb BE: be); log-likelihood value - 1971.4999; пример: Brown will probably be involved in passing it on to other sites as well as to colleagues at the Morgan Grenfell operation (BNC, 2021). / Браун, вероятно, будет участвовать в передаче его другим сайтам, а также коллегам по предприятию Morgan Grenfell. На пятом месте - глаголы различной семантики в форме инфинитива (the infinitive form of lexical verbs (e.g. forget, send, live, return)), как правило, это правые коллокаты, при этом левым коллокатом выступает модальный глагол; log-likelihood value - 1803.5964; пример: If the glider stalls on the launch it will probably drop a wing and may spin (BNC, 2021). / Если планер заглохнет при запуске, он, вероятно, уронит крыло и может вращаться.

Приведем значения нормированных частот употребления наречия possibly в разных функциональных стилях: художественная литература (243), научная литература (78), газетно-информационный стиль (52). Для наречия possibly наиболее частотными коллокатами являются как левые коллокаты, так и правые коллокаты. На первом месте также идут левые коллокаты, выраженные модальными глаголами, наиболее частотный could (modal auxiliary verb (e.g. will, would, can, could, 'll, 'd)); 2047.9735; пример: But, he pointed out, no one could possibly conceive what life would be like after the death of images (BNC, 2021). / Но, отметил он, никто не мог представить себе, какой будет жизнь после смерти изображений. На втором месте идут левые коллокаты, выраженные сочинительными союзами (coordinating conjunction (e.g. and, or, but)); 1518.2671; примep: Nowadays appreciations are often condensed, perhaps into a catalogue introduction, or possibly fitted into the preface for a book of illustrations (BNC, 2021). / В настоящее время оценки часто концентрируются, возможно, во введении в каталог или, возможно, в предисловии к книге иллюстраций. На третьем месте идут левые коллокаты, выраженные отрицательной частицей not (the negative particle not or n't); 1364.6864; пример: You can't possibly hope to get that in your early days as a student (BNC, 2021). / Вы не можете надеяться получить это в первые годы учебы. На четвертом месте идут правые коллокаты, выраженные глаголами различной семантики в начальной форме (the infinitive form of lexical verbs (e.g. forget, send, live, return)), при этом левыми коллокатами выступают модальные глаголы; 1023.8517; пример: But, he pointed out, no one could possibly conceive what life would be like after the death of images (BNC, 2021). / Но, отметил он, никто не мог представить себе, какой будет жизнь после смерти изображений. И на пятом месте - глагол to have в инфинитиве (the infinitive form of the verb HAVE: have), при этом правым коллокатом выступают модальные глаголы, а сама конструкция представляет собой конструкцию Modal Verb + Perfect Infinitive; 780.8865; пример: He cannot possibly have played better despite tiring in the second half of a patchy but exciting final (BNC, 2021). / Невозможно было сыграть лучше, несмотря на усталость во второй половине неоднозначного, но захватывающего финала.

Приведем значения нормированных частот употребления наречия perhaps в разных функциональных стилях: художественная литература (558), научная литература (336), газетно-информационный стиль (167). Для наречия perhaps характерны как правые, так и левые коллокаты. На первом месте - правые коллокаты, 
выраженные личными местоимениями (personal pronoun (e.g. I, you, them, ours)); 6067.9432; пример: Perhaps he could tell how he was, or where? / Может быть, он сможет сказать, как он себя чувствует или где? На втором месте - знаки препинания (punctuation: general separating mark - i.e. . , ! , : ; - or ?): в данном случае наречие используется в функции представления добавочной информации в предложении, как способ выражения модальности всего предложения, в эллиптических предложениях и как отдельное предложение; 5756.5192; пример: They nevertheless will have seen some, perhaps all the works included, and can thus comment usefully on the artist's standing, aims and achievements (BNC, 2021). / Тем не менее, они увидят некоторые, а может быть, и все работы, и, таким образом, могут дать полезные комментарии о статусе, целях и достижениях художника. На третьем месте - левые коллокаты, выраженные сочинительными союзами (coordinating conjunction (e.g. and, or, but)); 3521.6817; пример: We agree to help and perhaps I can combine the two visits (BNC, 2021). / Мы согласны помочь и, возможно, я смогу совместить два визита. На четвертом месте - правые коллокаты, выраженные общими наречиями (general adverb: an adverb not subclassified as AVP or AVQ (e.g. often, well, longer (adv.), furthest)), самыми частотными является most/the most; 1575.7248; пример: Perhaps most typically that of the timber framed building (BNC, 2021). / Возможно, наиболее типично здание с деревянным каркасом. На пятом месте - глагол to be в 3 лице ед.ч. (the -s form of the verb BE: is, 's); 805; пример: And that is perhaps the way they all want it (BNC, 2021). / И, возможно, все они этого хотят.

Приведем значения нормированных частот употребления наречия тауbе в разных функциональных стилях: художественная литература (232), научная литература (12), газетно-информационный стиль (55). Для наречия тауbе характерны как правые, так и левые коллокаты. На первом месте - правые коллокаты, выраженные личными местоимениями (personal pronoun (e.g. I, you, them, ours)); 5701.9791; пример: 'Мауbe he's got a girl in there,' I thought (BNC, 2021). / «Может, у него там девушка?» - подумал я. На втором месте левые коллокаты, выраженные сочинительными союзами (coordinating conjunction (e.g. and, or, but)); 1423.3608; пример: One source of interest can be a description of where the interview has taken place, perhaps a studio, or maybe the artist's home (BNC, 2021). / Одним из источников интереса может быть описание того, где проходило интервью, возможно, студия или, может быть, дом художника. На третьем месте - знаки препинания (punctuation: general separating mark - i.e. . , ! , : ; - or ?), как правило, maybe используется как самостоятельное предложение; 927.9496; пример: Of course, I still need to catch up on that necessary office work, maybe tomorrow... (BNC, 2021). / Конечно, мне все еще нужно наверстать упущенное по этой необходимой офисной работе, может быть, завтра... На четвертом месте - правые коллокаты, кавычки при прямой речи (рunctuation: quotation mark - i.e. ' or "); 920.3121; пример: 'Maybe I should have stayed and fought?' (BNC, 2021). / «Может, мне следовало остаться и драться?» На пятом месте - правые и левые коллокаты, выраженные общими наречими (general adverb: an adverb not subclassified as AVP or AVQ (e.g. often, well, longer (adv.), furthest)); 364.8591; пример: Maybe just throw him out down the road here (BNC, 2021). / Может быть, просто выбрось его по дороге здесь.

Приведем значения нормированных частот употребления наречия presumably в разных функциональных стилях: художественная литература (32), научная литература (49), газетно-информационный стиль (17). Для наречия presumably характерны как правые, так и левые коллокаты. На первом месте - левые коллокаты, выраженные знаками препинания, как правило запятой или тире, для ввода уточняющей информации в предложение (punctuation: general separating mark - i.e. . , ! , : ; - or ?); 1089.8089; пример: Neto's, and Castro's, Angolans, presumably (BNC, 2021). / Нето и Кастро, ангольцы, предположительно. На втором месте левые коллокаты, выраженные знаками препинания «левая скобка» (punctuation: left bracket - i.e. ( or [) для ввода дополнительной информации; 220.1919; пример: Thus (presumably unfairly) the UK 'would be more affected by this part of the Directive than other states' (BNC, 2021). / Таким образом (предположительно, несправедливо), Великобритания «будет больше затронута этой частью Директивы, чем другие государства». На третьем месте - левые коллокаты, выраженные модальными глаголами (modal auxiliary verb (e.g. will, would, can, could, 'll, 'd)); 169.5823; пример: Arab peacemakers will presumably seek to find a successor to Muawad (BNC, 2021). / Предположительно, арабские миротворцы будут искать преемника Муавада. На четвертом месте левые коллокаты, выраженные подчинительными союзами (subordinating conjunction (e.g. although, when)); 150.4372; пример: Presumably if it didn't kill them it gave them a very nasty headache (BNC, 2021). / Предположительно, если он их не убил, у них сильно заболела голова. На пятом месте - правые коллокаты, выраженные личными местоимениями (personal pronoun (e.g. I, you, them, ours)); 77.0042; пример: Presumably he means 'mortise' gauge (BNC, 2021). / Предположительно он имел в виду «врезной» калибр.

Приведем значения нормированных частот употребления наречия apparently в разных функциональных стилях: художественная литература (81), научная литература (95), газетно-информационный стиль (63). Для наречия apparently характерны как правые, так и левые коллокаты. На первом месте - правые коллокаты, выраженные причастием прошедшего времени глаголов различной семантики (the past participle form of lexical verbs (e.g. forgotten, sent, lived, returned)); 842.3447; пример: The issue of Al-Fajr in which the article appeared was apparently approved by the Ministry of Interior (BNC, 2021). / Выпуск «Аль-Фаджр», в котором появилась статья, был, по всей видимости, одобрен Министерством внутренних дел. На втором месте - левые коллокаты, выраженные формой прошедшего времени глагола to have (the past tense form of the verb HAVE: had, 'd); 625.2263; пример: Indeed, the fact that he had apparently used an editor from a Manchester newspaper for some of his purposes was latched onto and quoted as a sign that he was 'suspect' and 'disloyal' (BNC, 2021). / Действительно, тот факт, что он явно использовал редактора манчестерской газеты для некоторых из своих целей, был зафиксирован и процитирован как признак того, что он был «подозреваемым» и «нелояльным». 
На третьем месте - левые коллокаты, выраженные глаголом to be в форме прошедшего времени (the past tense forms of the verb BE: was and were); 620.317; пример: After the March 1991 releases, over 70 detainees were still held and were apparently due to be brought to trial in April (BNC, 2021). / После освобождения в марте 1991 года более 70 задержанных все еще находились под стражей и, по всей видимости, должны были предстать перед судом в апреле. На четвертом месте - правые коллокаты, выраженные прилагательными общей семантики или прилагательными с положительной оценкой (adjective (general or positive) (e.g. good, old, beautiful)); 602.8498; пример: With the exception of a small group of public schools, there is the apparently simple distinction between state schools and Roman catholic schools which occurs in Britain (BNC, 2021). / За исключением небольшой группы государственных школ, существует очевидное простое различие между государственными школами и римскокатолическими школами, которое имеет место в Британии. На пятом месте - левые коллокаты, выраженные знаками препинания (punctuation: general separating mark - i.e. . , ! , : ; - or ?); 418.9538; пример: A World Apart is a 'truly golden' work, despite the presence in it, apparently, of a foul anti-Semitism (BNC, 2021). / A World Apart - «поистине золотое» произведение, несмотря на присутствие в нем, видимо, гнусного антисемитизма.

Приведем значения нормированных частот употребления наречия conceivably в разных функциональных стилях: художественная литература (1.73), научная литература (6), газетно-информационный стиль (1.78). Для наречия conceivably характерны как правые, так и левые коллокаты. На первом месте - левые коллокаты, выраженные модальными глаголами (modal auxiliary verb (e.g. will, would, can, could, 'll, 'd)); 669.7988; пример: But sheer pressure - from within, from the Soviet Union and other allies and from the West - could conceivably do the job (BNC, 2021). / Но явное давление - изнутри, со стороны Советского Союза и других союзников, а также со стороны Запада - вполне могло бы сработать. На втором месте - правые коллокаты, выраженные глаголом to be в инфинитиве, как правило, левым коллокатом являются модальные глаголы (the infinitive form of the verb BE: be); 176.3148: пример: Red Riding Hood could conceivably be taken in by an Irish Setter (BNC, 2021). / Красную Шапочку предположительно может забрать ирландский сеттер. На третьем месте - правые коллокаты, выраженные глаголами различной семантики, как правило, левым коллокатом при этом являются модальные глаголы (the infinitive form of lexical verbs (e.g. forget, send, live, return)); 176.3148; пример: Small genetic changes might conceivably aggravate their nuisance value (BNC, 2021). / Небольшие генетические изменения могут усугубить их неприятную ценность. На четвертом месте - правые коллокаты, выраженные глаголом to have в инфинитиве (the infinitive form of the verb HAVE: have), при этом левыми коллокатами являются модальные глаголы; 145.391; пример: Because it used not to understand markets, it cannot conceivably have rectified this gap in its political intelligence (BNC, 2021). / Поскольку раньше он не понимал рынков, он вряд ли мог исправить этот пробел в своем политическом интеллекте. На пятом месте - левые коллокаты, выраженные отрицательной частицей not с модальным глаголом (the negative particle not or n't); 30.0884; пример: Because it used not to understand markets, it cannot conceivably have rectified this gap in its political intelligence (BNC, 2021). / Поскольку раньше он не понимал рынков, он вряд ли мог исправить этот пробел в своем политическом интеллекте.

Приведем значения нормированных частот употребления наречия plausibly в разных функциональных стилях: художественная литература (0.37), научная литература (5.45), газетно-информационный стиль (0.21). Для наречия plausibly характерны как правые, так и левые коллокаты. На первом месте - правые коллокаты, выраженные глаголом to be в форме инфинитива (the infinitive form of the verb BE: be), при этом левый коллокат выражен модальным глаголом; 192.9602; пример: This might plausibly be dismissed as 'Victorian stupidity' were it not for the tact that in Britain in 1988 a similar view was actually inscribed in law (BNC, 2021). / Это можно было бы с правдоподобием отклонить как «викторианскую глупость», если бы не тактичность, которая в Великобритании в 1988 году фактически была закреплена в законе. На втором месте - левые коллокаты, выраженные модальными глаголами (modal auxiliary verb (e.g. will, would, can, could, 'll, 'd)), самые частотные - can, could, might; 141.2069; пример: This might plausibly be dismissed as ‘Victorian stupidity' were it not for the tact that in Britain in 1988 a similar view was actually inscribed in law (BNC, 2021). / Это можно было бы с правдоподобием отклонить как «викторианскую глупость», если бы не тактичность, которая в Великобритании в 1988 году фактически была закреплена в законе. На третьем месте - правые коллокаты, выраженные причастиями прошедшего времени глаголов различной семантики, как правило в пассивном залоге (the past participle form of lexical verbs (e.g. forgotten, sent, lived, returned)); 63.3947; пример: Poems are more plausibly referred to as works of art than novels; the latter may have obviously aesthetic qualities, but have many other things in them as well (BNC, 2021). / Стихи более правдоподобно относят к произведениям искусства, чем к романам; последние могут иметь явно эстетические качества, но также иметь в себе много других вещей. На четвертом месте - левые коллокаты, выраженные глаголом to bе в форме причастия прошедшего времени, как правило с использованием времени Present Perfect Tense в пассивном залоге (the past participle form of the verb BE: been); 145.391; пример: Such theories have been plausibly criticized for their romantic and Utopian strains, also for the way they echo and sometimes invoke a post-Freudian version of the polymorphous perverse (BNC, 2021). / Такие теории правдоподобно критиковались за их романтические и утопические черты, а также за то, как они перекликаются с постфрейдистской версией полиморфно-перверсивной теории, а иногда и ссылаются на нее. На пятом месте - левые коллокаты, выраженные общими наречиями (general adverb: an adverb not subclassified as AVP or AVQ (see below) (e.g. often, well, longer (adv.), furthest)); 33.8533; пример: No less plausibly than Attlee and his colleagues in 1945, Mrs Thatcher was entitled to declare: 'We are the masters now' (BNC, 2021). / Не менее правдоподобно, чем Эттли и его коллеги в 1945 году, миссис Тэтчер имела право заявить: «Теперь мы хозяева». 
В результате анализа можно заключить следующее. С точки зрения семантики большую степень уверенности выражают модусные наречия probably, presumably, apparently и plausibly; меньшая степень уверенности, сомнения выражены наречиями perhaps, maybe, conceivably, possibly. С точки зрения частотности использования в речи наиболее используемые наречия в художественной литературе представлены наречиями perhaps (558); probably (268); possibly (243); maybe (232); conceivably (173); наиболее используемые наречия в научном стиле представлены наречиями perhaps (336); probably (243); apparently (95); самые используемые наречия в газетно-информационном стиле представлены perhaps (167); probably (165); apparently (63). C точки зрения частеречных сочетаемостных возможностей данных наречий можно заключить следующее: практически все эти наречия (probably, possibly, presumably, plausibly, conceivably) имеют высокую степень сочетаемостных возможностей с модальными глаголами; с этой точки зрения данные наречия с большой долей вероятности могут быть использованы для выражения модуса всей пропозиции. Наречия perhaps и тауbe также с большой долей вероятности могут быть употреблены для выражения модуса всей пропозиции. Синтагматически это выражается в их способности быть обособленными знаками препинания (скобки, запятые), при этом данные наречия могут вводить в предложение добавочную или уточняющую информацию. Наиболее частотные модели употребления данных наречий связаны с глаголами: to be, to have и другими глаголами различной семантики, которые выступают в подавляющем большинстве случаев (80\%) как правые коллокаты, при этом, как правило, левые коллокаты выражены модальными глаголами. Стоит также отметить, что для некоторых наречий (possibly, perhaps, maybe) характерна модель использования с сочинительными союзами (and, but), для наречия presumably характерно употребление с подчинительными союзами (although и др.) в постпозиции. Отметим, что для наречия apparently характерны следующие модели, которые не свойственны другим наречиям: модели использования в прошедшем времени, конструкция Modal Verb + Perfect Infinitive и как модификатор прилагательных. В качестве выражения признаковости другого наречия могут использоваться наречия perhaps, maybe, plausibly. При выражении отрицания предикативной группой предложения более характерно использование наречий possibly, conceivably. С личными местоимениями в инициальной позиции более характерно употребление наречий maybe, presumably.

\section{Заключение}

Такие исследования, в фокусе которых находится выявление закономерностей употребления языковых средств в различных коммуникативных сферах, несомненно представляют эвристическую ценность для перевода. Знание закономерностей функционирования языковых единиц в речи и типичных, наиболее частотных моделей их употребления очень важно на втором этапе переводческого семиозиса, т.е. при перевыражении подлинника, особенно при переводе на неродной язык. Такой подход стал возможен благодаря развитию и инкорпорированию методов корпусной лингвистики и интернет-технологий в теорию и практику перевода и сопоставительные исследования.

В данной статье был проведен анализ модусных наречий в английском языке с точки зрения закономерностей их использования в речи. Исследование проведено на базе Британского национального корпуса. Выявлены типичные, регулярные модели употребления данных наречий в различных функциональных стилях речи.

Перспективы дальнейшего исследования мы видим в более детальном изучении языковой репрезентации логико-семантических категорий в сопоставительном аспекте с использованием методов корпусной лингвистики для оптимизации поиска вариантных соответствий в решении переводческих задач.

\section{Источники | References}

1. Захаров В. П., Богданова С. Ю. Корпусная лингвистика. СПб.: Изд-во Санкт-Петербургского университета, 2020.

2. Крюков А. Н. Методологические основы интерпретативной концепции перевода: автореф. дисс. ... д. филол. н. M., 1988.

3. Крюков А. Н. Теория перевода. М.: Военный институт, 1989.

4. Швейцер А. Д. Контрастивная стилистика. М., 1993.

5. Bowker L. A Corpus-Based Approach to Evaluating Student Translations // Translator. 2000. Vol. 6. Iss. 2.

6. British National Corpus (BNC). 2021. URL: http://bncweb.lancs.ac.uk/

7. Bruti S. Corpus Approaches and Audiovisual Translation // The Palgrave Handbook of Audiovisual Translation and Media Accessibility / ed. by Ł. Bogucki, M. Deckert. Cham: Palgrave Macmillan, 2020.

8. Buts J., Jones H. From Text to Data: Mediality in Corpus-Based Translation Studies // MonTI. Monografías De Traducción E Interpretación. 2021. Vol. 13.

9. Corpus-Based Research in Legal and Institutional Translation: Special Issue / ed. by F. P. Ramos // Translation Spaces. 2019. Vol. 8. Iss. 1.

10. De Sutter G., Cappelle B., De Clercq O., Loock R., Plevoets K. Towards a Corpus-based, Statistical Approach to Translation Quality: Measuring and Visualizing Linguistic Deviance in Student Translations // Linguistica Antverpiensia. New Series - Themes in Translation Studies. 2018. Vol. 16.

11. Doval I., Sánchez Nieto M. T. Parallel Corpora for Contrastive and Translation Studies. New Resources and Applications. Amsterdam: John Benjamin Publishing Company, 2019. 
12. Edina R., Götz A., Pataky E. Translation Studies and Corpus Linguistics: Introducing the Pannonia Corpus // Acta Universitatis Sapientiae. Philologica. 2018. Vol. 9. Iss. 3.

13. Garcia A. F. Can a Corpus-Driven Lexical Analysis of Human and Machine Translation Unveil Discourse Features that Set Them Apart? 2021. URL: https://benjamins.com/catalog/target.20065.fra

14. Ji M., Oakes M., Defeng L., Hareide L. Corpus Methodologies Explained an Empirical Approach to Translation Studies. L.: Routledge, 2018.

15. Mikhailov M., Cooper R. Corpus Linguistics for Translation and Contrastive Studies. Routledge Corpus Linguistics Guides. L.: Routledge, 2016.

16. Oakes M. P., Ji M. Quantitative Methods in Corpus-Based Translation Studies. A Practical Guide to Descriptive Translation Research. Amsterdam: John Benjamin Publishing Company, 2012.

17. Oxford English Dictionary. 2021. URL: https://lexico.com

18. Pastor G. C., Seghiri M. Corpus-Based Approaches to Translation and Interpreting. From Theory to Applications. Frankfurt am Main: PeterLang Edition, 2016.

\section{Информация об авторах | Author information}

RU Абдульманова Аделя Хамитовна ${ }^{1}$, к. филол. н., доц. Куликова Марина Николаевна ${ }^{2}$, к. филол. н.

Лекомцева Ирина Алексеевна ${ }^{3}$, к. филол. н.

1,2, 3 Санкт-Петербургский государственный университет

EN Abdulmanova Adelia Khamitovna ${ }^{1}, \mathrm{PhD}$

Kulikova Marina Nikolaevna ${ }^{2}, \mathrm{PhD}$

Lekomtseva Irina Alekseevna ${ }^{3}, \mathrm{PhD}$

$1,2,3$ St. Petersburg State University

${ }^{1}$ aahvsp@rambler.ru, ${ }^{2}$ kulikm@yandex.ru, ${ }^{3}$ rainbow5@mail.ru

\section{Информация о статье | About this article}

Дата поступления рукописи (received): 12.10.2021; опубликовано (published): 30.11.2021.

Ключевые слова (keywords): модусные наречия; корпусные методы в переводоведении; перевод как речевая деятельность; выбор языковых средств; сопоставительные исследования; modus adverbs; corpus research methods in translation studies; translation as speech activity; choice of linguistic means; comparative studies. 